\title{
Clarification of the Five-Factor Model With the Abridged Big Five Dimensional Circumplex
}

\author{
John A. Johnson and Fritz Ostendorf
}

\begin{abstract}
This article shows how the Abridged Big Five Dimensional Circumplex (AB5C; W. K. Hofstee, B. de Raad, \& L. R. Goldberg, 1992) clarifies disputes about the Big Five or five-factor model. Trait ratings from instruments representing 4 versions of the Big Five (L. R. Goldberg, 1992; R. Hogan \& J. A. Johnson, 1981; R. R. McCrae \& P. T. Costa, 1985b. 1987; W. T. Norman.1963) were subjected to separate AB5C analyses for 2,148 American and 1,285 German Ss. Replicated results formed standard designation codes representing trait adjectives' primary and secondary factor loadings. These codes unveiled the unique coloring imparted by secondary loadings to different scales proffered by researchers to represent the 5 factors.
\end{abstract}

Recent reviews (Digman, 1990; John, 1990a, 1990b; McCrae \& John, 1992) have indicated that the universe of personality traits can be represented at an abstract level by five broad dimensions: (Factor I) Extraversion, (Factor II) Agreeableness, (Factor III) Conscientiousness, (Factor IV) Emotional Stability, and (Factor V) Intellect or Openness. AIthough the clear intent of these reviews is to stress commonality, consensus, and agreement of thinking about the Big Five or five-factor model (FFM), a prominent section of each review discusses disagreements concerning the precise nature of, and best labels for, the five factors. This article describes how the Abridged Big Five Dimensional Circumplex (AB5C; Hofstee, de Raad, \& Goldberg, 1992) might clarify these disputes.

A truism of factor analysis is that the nature of the factors is determined by the content of the variables entered into the analysis (Guilford, 1975). Obviously, if a personality inventory lacks items that assess agreeableness, an Agreeableness factor will not emerge in a factor analysis of its items or scales (see Lanning \& Gough, 1991; McCrae, Costa, \& Piedmont, 1993). A

John A. Johnson, Department of Psychology, Pennsylvania State University, DuBois Campus; Fritz Ostendorf, Department of Psychology, University of Koblenz-Landau, Federal Republic of Germany.

The research described in this article was conducted while John A. Johnson was a visiting research fellow at Universität Bielefeld, Bielefeld, Germany, supported by a fellowship from the Alexander von Humboldt-Stiftung. Gratitude is expressed to Alois Angleitner and Robert Wicklund for helping to arrange John A. Johnson's stay and to the psychology department at Bielefeld and the von Humboldt-Stiftung for their support.

We thank Lewis R. Goldberg, Willem K. B. Hofstee. Robert Hogan, R. R. McCrae, and the anonymous reviewers for their feedback on drafts of this article.

Correspondence concerning this article should be addressed to John A. Johnson, Pennsylvania State University, College Place, DuBois, Pennsylvania 15801. Electronic mail may be sent to j5j@psuvm (Bitnet) or j5j@psuvm.psu.edu. (Internet). more subtle application of the truism, however, concerns the secondary loadings of the variables defining a factor. For example. Factor $\mathrm{V}$ in one analysis might be defined by many items that have a secondary positive loading on Factor III: the interpretation of this factor will be different from a second analysis in which Factor $V$ is defined by many items with a secondary loading on Factor I.

Hofstee et al. (1992) hypothesized that differences in secondary factor loadings may have contributed to the equivocal interpretation of the Big Five factors across research programs. The present study tests this hypothesis by using Hofstee et al.'s AB5C model, which explicitly takes into account secondary factor loadings. Trait rating data from instruments representing four versions of the Big Five (Goldberg, 1992; Hogan \& Johnson, 1981; McCrae \& Costa, 1985b, 1987; Norman. 1963) were subjected to separate $A B 5 C$ analyses. Results that replicated across a plurality of data sets formed a standard AB5C designation code (e.g., in most data sets perceptive loaded primarily on $\mathrm{V}$ and secondarily on III for a V+III+ designation; liberal loaded primarily on $\mathrm{V}$ and secondarily on I for a $\mathrm{V}+\mathrm{I}+$ designation). The standard designation codes allowed a comparison of the "secondary flavor" of different scales proffered by researchers to represent the five factors.

These analyses were expected, first, to help resolve disputes about where specific personality constructs belong within the FFM and, second, to help resolve controversy about the nature of the five factors themselves. Some of these disputed constructs include positive emotions, conformity, ambition, impulsivity, and intellect.

Positive emotions have been identified as part of Factors I and II. In Costa and McCrae's (1985, 1992) NEO model, Warmth (affection and friendliness) and Positive Emotions are facets of Extraversion (Factor I), and Watson and Clark (in press) argued that positive emotionality lies at the core of this factor. On the other hand, Goldberg (1992) and Hogan and Johnson (1981) suggested that terms reflecting prosocial, positive emotions (warm, kind, and empathic) define what makes a person agreeable (Factor II). 
Agreeable means not only being amiable, congenial, and friendly but also acquiescent, amenable, and compliant (Costa, McCrae, \& Dye, 1991). Thus, Costa et al. (1991) suggested that terms denoting conformity and compliance belong to the Agreeableness (Factor II) domain. In another view, conformity to social expectations and norms has been regarded as central to the Factor III domain by those who have studied moral development, delinquency, and criminality (Hogan, Johnson, \& Emler, 1978; Johnson, 1983; Laufer, Johnson, \& Hogan, 1981). Finally, conformity has also been linked with the negative pole of Factor $\mathrm{V}$ through research demonstrating that persons open to experience tend to be nonconformists (McCrae \& Costa, 1985a).

McCrae and John (1992) noted that "the term ambitious may mean wanting to get ahead or wanting to get things done. The former is probably an aspect of [I], the latter an aspect of [III]" (p. 196). Hogan (1986; Hogan \& Johnson, 1981) was unique among advocates of the FFM in choosing to create separate Factor I scales for ambition and simple sociability. Goldberg (1992) and McCrae and Costa (1985b) assigned the terms hardworking and ambitious to Factor III.

Impulsivity has proved to be a complex, multifaceted construct and therefore particularly difficult to place within the FFM (McCrae \& Costa, 1985a). It was originally linked with Extraversion (I+) by Eysenck (Eysenck \& Eysenck, 1977) until he decided to move it to his Psychoticism (a combination of IIand III $\rightarrow$ ) dimension (John, 1990a). Hogan (1986; Hogan \& Johnson, 1981) located impulsivity at the low end of his Factor III Prudence scale, whereas Costa and McCrae identified impulsivity as a facet of their Neuroticism (IV-) scale.

The placement of intellect-related terms such as intelligent, intellectual, and perceptive seems to be a function of a researcher's conceptualization of Factor V. Those who conceive of Factor V as an intellect factor (Goldberg, 1992; Hogan, 1986) naturally assign these terms to that factor. Those who see Factor $\mathrm{V}$ as Openness to Experience (McCrae \& Costa, 1985b) perceive these terms as belonging to the achievement-striving syndrome of Factor III.

As might be expected, disputes about the location of specific personality terms are related to controversies concerning the interpretation of the broader Big Five. The analyses conducted in the present study, therefore, were expected to shed light on several controversies concerning the interpretation of these factors (Digman, 1990; McCrae \& John, 1992). The first problem of interpretation concerns whether the "agreeableness" of Factor II refers primarily to a tendency to agree with others or to a pleasant disposition. A second issue is whether the core of Factor III is inhibitory impulse control or organized purposefulness. Perhaps the thorniest controversy is over Factor V, which has been alternatively interpreted as Intellect and as Openness to Experience.

\section{Method}

\section{Data Sets}

The current study reanalyzes data collected in previous studies; thus, a complete description of the subjects and measures can be found in the original sources. Table 1 summarizes some characteristics of the data sets. In each of these data sets, rating scales anchored by bipolar personality terms (e.g., anxious-calm) had been constructed for the specific purpose of assessing each factor of the FFM. These ratings were factor analyzed to yield five varimax-rotated principal components. The $\mathrm{AB} 5 \mathrm{C}$ analyses in the present study began with these matrices.

Because the personality terms in the present study are bipolar, all statements concerning these terms imply a converse statement about the term's opposing anchor. For example, stating that extraverted is a $\mathrm{H}+\mathrm{I}+$ term implies that introverted is a $\mathrm{I}-\mathrm{I}-$ ter $\mathrm{m}$. With a few exceptions, this article reports findings in terms of the positively keyed end of each bipolar scale. In some cases, different researchers have chosen different opposing anchors (e.g., talkative-silent vs. talkative-quiet). Readers desiring to know which anchors are used by which researchers should consult the original sources.

\section{Analyses of Personality Terms}

A FORTRAN program (available from either author) was written to postmultiply the rotated matrices from the previous data sets by the submatrices described by Hofs tee et al. (1992). This generated loadings of each personality term on factors inserted every $30^{\circ}$ within each of the 10 circumplexes formed by all combinations of the five factors taken two at a time. (See Hofstee et al. for the rationale behind partitioning the circumplex "pie" into $30^{\circ}$ "slices.") The program locates each term's highest loading among the 90 factor poles and produces the label for that personality term in the "AB5C language" coined by Hofstee et al. For example, the highest loading for analytical in most of the data sets was found in the circumplex defined by Factor III $(x-$ axis $)$ and Factor $V(y-a x i s)$ on the factor inserted at $60^{\circ}$. This term therefore received a V+III+ (primarily Factor $V$ and secondarily Factor III) designation.

After all personality terms in each data set received an AB5C designation, these designations were compared across data sets to determine a consensus or composite $\mathrm{AB} 5 \mathrm{C}$ designation. For exa mple, analytical (analytisch in German) received a V+III+ designation in one of the two American samples that completed the Goldberg (1992) inventory and a V+II-designation in the other American sample that completed this inventory, a V+III+ designation in both German samples that completed the Goldberg inventory, a $\mathrm{V}+\mathrm{III}+$ by the Americans who completed the McCrae and Costa (1985b) inventory, a III+V+ by one of the German samples that completed the McCrae and Costa inventory, and a V+III+ by the other German sample that completed this inventory. Thus, analytical clearly appears to be a V+III+ personality term, regardless of the inventory in which it is embedded or even of the language spoken by the subjects.

Composite AB5C codes were calculated for 98 (72\%) of the 137 terms in the manner described above (i.e., by finding the most common AB5C code across samples). An additional 23 (17\%) composite AB5C codes were calculated by following the procedure of Hofstee et al. (1992, p. 161), whereby two points are assigned for the primary and one point for the secondary part of the designation of the term under consideration. The composite designation in $\mathrm{AB} 5 \mathrm{C}$ space is simpiy the unweighted sum of the designations of the term across samples.

The analyses described above yielded A B5C depictions of personality terms that transcend, to some degree, the theoretical assumptions of the authors of each set of rating scales. Because the authors of the rating scales each held somewhat unique conceptions of the FFM, they chose different sets of personality terms and occasionally assigned the terms, a priori, to different factors (e.g., Goldberg, 1992, assigned intelligent to Factor V, whereas McCrae and Costa, 1985b, assigned this term to Factor III). The construction of composite AB5C depictions, on the basis of multiple samples responding to inventories based on different conceptions of the FFM, was expected to help neutralize 
Table 1

Data Sets Reanalyzed for the Present Study

\begin{tabular}{|c|c|c|c|}
\hline Reference & Instrument & $N$ & Source of ratings \\
\hline Goldberg (1992) ${ }^{\mathbf{a}}$ & Markers for the Big Five & 157 & Self-ratings \\
\hline Goldberg (1992) ${ }^{\mathbf{a}}$ & Markers for the Big Five & 175 & Self-ratings \\
\hline Johnson (in press) & Bipolar adjective rating scales & 156 & Self-ratings \\
\hline Johnson (in press) ${ }^{b}$ & Bipolar adjective rating scales & 466 & Peer ratings \\
\hline McCrae and Costa (1987) & Goldberg items ${ }^{\mathrm{c}}$ & 738 & Peer ratings \\
\hline Norman (1963) & Peer nomination rating scales & 215 & Peer ratings ${ }^{d}$ \\
\hline Norman (1963) & Peer nomination rating scales & 245 & Peer ratings ${ }^{d}$ \\
\hline Ostendorf (1990) & Markers for the Big Five ${ }^{e}$ & 401 & Self-ratings \\
\hline Ostendorf (1990) & Markers for the Big Five ${ }^{e}$ & 502 & Peer ratings \\
\hline Johnson (in press) & Bipolar adjective rating scales ${ }^{c}$ & 183 & Self-ratings \\
\hline Johnson (in press) & Bipolar adjective rating scales ${ }^{e}$ & 96 & Peer ratings \\
\hline Ostendorf (1990) & Goldberg items ${ }^{e}$ & 401 & Self-ratings \\
\hline Ostendorf (1990) & Goldberg items" & 502 & Peer ratings \\
\hline Ostendorf (1990) & Peer nomination rating scales ${ }^{e}$ & 401 & Self-ratings \\
\hline Ostendorf (1990) & Peer nomination rating scales ${ }^{e}$ & 502 & Peer ratings \\
\hline
\end{tabular}

Note. All samples are traditionally aged college students except for McCrae and Costa's (1987) participants in the Augmented Baltimore Longitudinal Study of Aging (average age $=60$ for men and 54 for women) and Ostendorfs (1990) recruits from a newspaper advertisement (average age $=33$ ).

${ }^{2}$ Factor loadings were obtained from an earlier unpublished version of the Goldberg (1992) article. The first sample is described in Study 1 and the second sample in Study 3 of the published article. The 50-item standard marker inventories shared 46 of the same items. ${ }^{b}$ A published description of scales can be found in Johnson. Germer, Efran, and Overton (1988). " Assembled from items reported in Goldberg (1980) and Goldberg (1983). 'Norman's (1963) peer nomination technique. involving nominations from 4 to 10 peers within groups ranging from 6 to 16 acquaintances. differs from the straightforward Likert ratings used in the other data sets. The $N$ s reported here refer to the number of ratees: the number of ratings provided is somewhere between 4 and 10 times these reportcd $N$ s. The Norman scales also differ from the others in that his scales were anchored by elaborate descriptions containing several trait terms. The terms reported here are those chosen by Norman to represent the longer descriptions. "German translation of the instrument.

both random fluctuations in factor loadings and the theoretical expectations of the inventory authors.

Doubtlessly, designations for some terms (e.g., imaginative) are more "theory neutral" than others (e.g., enthusiastic) because the former term appears on all four inventories, whereas the latter appears only on one. Furthermore, agreement on $\mathrm{AB} 5 \mathrm{C}$ depictions was stronger for some terms, like fair (II+IIIt in every sample) than others, such as energetic (alternatively depicted as $\mathrm{I}+\mathrm{II}+, \mathrm{I}+\mathrm{III}+, \mathrm{I}+\mathrm{V}+, \mathrm{II}+\mathrm{I}+$, and IV+I+ in different samples). In $16(12 \%)$ of the cases, no consensus was found for the secondary portion of the $\mathrm{AB} 5 \mathrm{C}$ code, requiring a somewhat subjective decision on the final $A B 5 C$ depiction. Within these limitations, however, these analyses provide a depiction of personality terms that is more reliable and theory neutral than depictions derived from a single inventory administered to a single sample. ${ }^{1}$

It was predicted that disagreements in the literature about the assignment of personality terms to factors would occur for terms depicted by $\mathrm{AB} 5 \mathrm{C}$ blends (e.g... cheerful as $\mathrm{I}+\mathrm{II}+$ ) rather than for terms with A B5C factor-pure depictions (e.g., ialkative as $\mathrm{I}+\mathrm{I}+$ ). If a term referring to positive emotions (such as cheerful) received a I+II+ or II+I+ designation, this would explain why some researchers claim that positive emotions belong to the Agreeableness domain (Factor II), whereas others assert that they belong to the domain of Extraversion (Factor I).

\section{Analyses of Personality Scales}

The aforementioned anal:ses provided a relatively theory-neutral language for talking about personality terms. After establishing $\mathrm{AB} 5 \mathrm{C}$ depictions of all terms, we returned to each of the inventories to assess the A B5C character of the authors' scales. Again, Hofstee et al.'s (1992, p. 161) method of assigning two points for the primary and one point for the secondary part of the designation of each term was used. The scale designation in A B5C space was defined as the unweighted sum of the designations of each term on that scale. The scale was considered factor pure (i.e., I+I+, II+II+, etc.) only if it contained a plurality of factor-pure terms.

For example, for the six terms on Hogan and Johnson's (1981) Factor III scale, three terms were III + II + , one term was III+III+, one term was $\mathrm{III}+\mathrm{IV}+$, and one term was III+V-. Overall, this yielded 13 points for $\mathrm{III}+, 3$ points for IIt, and $\mathrm{I}$ point each for IVt and $\mathrm{V}-$. Thus, the overall AB5C depiction for this scale was III+II+. In contrast, the Norman (1963) III scale contained two III+III+ terms, a III+II+ term and a III + IV+ term for an overall III+III+ designation.

It was precicted that disagreements of factor interpretation between research groups would be found in cases where the different research groups lised scales depicted by different $A B 5 C$ blends. If one author's Factor $\mathrm{V}$ scale received an overall $\mathrm{V}+\mathrm{l}+$ designation, this might explain how the author conceptualized the Factor $V$ domain differently from an author whose Factor $\mathrm{V}$ scale received a V+III+ designation.

(text continues on page 570)

\footnotetext{
1 Alternatively, composite AB5C codes could be computed by applying the $\mathrm{AB} 5 \mathrm{C}$ algorithm to the average principal-component values found in Table 2. Results from this alternative method differ slightly from those obtained with the present method, particularly in cases where average secondary loadings are similar in magnitude or when the standard deviations of the loadings are relatively high. Persons desiring to explore this or other methods of data analysis can request an electronic copy of the original principal-components matrices from John A. Johnson.
} 
Table 2

Composite ABSC Depictions for all Personality Terms

\begin{tabular}{|c|c|c|c|c|c|c|c|c|c|c|c|c|c|c|}
\hline \multirow[b]{3}{*}{ Facet } & \multirow[b]{3}{*}{ Term } & \multirow[b]{3}{*}{ Facet } & \multirow[b]{3}{*}{ Anchors } & \multirow{3}{*}{$\begin{array}{l}N \text { of } \\
\text { data } \\
\text { sets }\end{array}$} & \multicolumn{10}{|c|}{ Factor loadings across data sets ${ }^{\mathrm{a}}$} \\
\hline & & & & & \multicolumn{2}{|c|}{ Factor I } & \multicolumn{2}{|c|}{ Factor II } & \multicolumn{2}{|c|}{ Factor III } & \multicolumn{2}{|c|}{ Factor IV } & \multicolumn{2}{|c|}{ Factor V } \\
\hline & & & & & $M$ & $S D$ & $M$ & $S D$ & $M$ & $S D$ & $M$ & $S D$ & $M$ & $S D$ \\
\hline & & & & Factor I & & & & & & & & & & \\
\hline \multirow[t]{6}{*}{$\mathbf{I}+\mathbf{I}+$} & Extraverted & $\mathbf{I}-\mathbf{I}-$ & Introverted & 6 & .71 & .07 & .03 & .09 & -.02 & .14 & .08 & .08 & -.03 & .08 \\
\hline & Frank, open & & Secretive & 4 & .77 & .02 & .07 & .12 & -.06 & .10 & .04 & .06 & .10 & .07 \\
\hline & Fun Ioving & & Sober & 3 & .57 & .03 & .21 & .06 & -.19 & .04 & .06 & .05 & .23 & .10 \\
\hline & Sociable & & $\begin{array}{l}\text { Retiring, reclusive, unsociable, } \\
\text { or solitary }\end{array}$ & 15 & .76 & .11 & .13 & .09 & -.05 & .06 & .11 & .09 & .02 & .09 \\
\hline & Straightforward & & Manipulative & 3 & .32 & .23 & .16 & .27 & .12 & .17 & .02 & .12 & -.01 & .01 \\
\hline & Talkative & & Silent or quiet & 15 & .76 & .08 & -.03 & .10 & -.01 & .06 & .01 & .08 & .02 & .07 \\
\hline \multirow[t]{5}{*}{$\mathrm{I}+\mathrm{II}+$} & Aflectionate & I-II- & Reserved & 3 & .61 & .06 &. .44 & .16 & -.01 & .12 & .07 & .06 & .12 & .03 \\
\hline & Cheerful & & Serious or depressed & 7 & .44 & .14 & .26 & .13 & .03 & .17 & .38 & .21 & .02 & .08 \\
\hline & Enthusiastic & & Unenthusiastic & 2 & .60 & .06 & .38 & .13 & .07 & .22 & .03 & .06 & .11 & .10 \\
\hline & Friendly & & Aloof & 3 & .55 & .07 & .50 & .07 & .11 & .05 & .14 & .02 & .05 & .03 \\
\hline & Joiner & & Loner & 3 & .52 & .01 & .24 & .10 & -.04 & .14 & -.02 & .14 & -.13 & .05 \\
\hline $\mathrm{I}+\mathrm{II}-$ & Dominant & $\mathrm{I}-\mathrm{II}+$ & Submissive & 5 & .47 & .16 & -.37 & .12 & .25 & .06 & .14 & .05 & .22 & .03 \\
\hline \multirow[t]{5}{*}{ I+III+ } & Active & I-III- & Passive or inactive & 11 & .61 & .09 & .04 & .16 & .32 & .11 & .13 & .14 & .11 & .11 \\
\hline & Energetic & & Unenergetic or leisurely & 11 & .52 & .11 & .13 & .13 & .40 & .19 & .13 & .13 & .05 & .11 \\
\hline & Enterprising & & Unambitious & 4 & .42 & .14 & .06 & .22 & .39 & .15 & .18 & .11 & .17 & .19 \\
\hline & Self-confident & & Unassured & 1 & .68 & .00 & .12 & .00 & .22 & .00 & .14 & .00 & .09 & .00 \\
\hline & Vigorous & & Unenergetic & 4 & .56 & .12 & .10 & .09 & .40 & .07 & .17 & .22 & .04 & .12 \\
\hline $\mathrm{I}+\mathrm{III}-$ & Person oriented & $\mathrm{I}-\mathrm{III}+$ & Task oriented & 3 & .36 & .00 & .26 & .08 & -.34 & .04 & -.12 & .14 & .08 & .10 \\
\hline \multirow[t]{7}{*}{$\mathrm{I}+\mathrm{IV}+$} & Adventurous & I-IV- & Unadventurous or cautious & 5 & .53 & .25 & -.06 & .16 & -.30 & .25 & .22 & .14 & .08 & .07 \\
\hline & Assertive & & Unassertive & 1 & .59 & .00 & -.03 & .00 & .21 & .00 & .26 & .00 & .22 & .00 \\
\hline & Bold & & Timid & 11 & .47 & .18 & -.10 & .17 & .04 & .12 & .30 & .15 & .18 & .11 \\
\hline & Comfortable & & Self-conscious & 3 & .40 & .10 & .20 & .07 & .13 & .04 & .45 & .12 & .11 & .07 \\
\hline & Not lonely & & Lonely & 3 & .44 & .13 & .08 & .06 & .09 & .07 & .39 & .09 & .04 & .05 \\
\hline & Outgoing & & Reserved & 4 & .76 & .03 & .10 & .07 & -.11 & .10 & .16 & .10 & .01 & .13 \\
\hline & Self-assured & & Shy & 4 & .41 & .30 & .03 & .07 & .03 & .10 & .46 & .20 & .11 & .09 \\
\hline \multirow[t]{8}{*}{$\mathrm{I}+\mathrm{V}+$} & Daring & $\mathrm{I}-\mathrm{V}-$ & Unadventurous & 3 & .43 & .11 & -.11 & .05 & -.12 & .18 & .24 & .09 & .29 & .23 \\
\hline & Forceful & & Submissive & 1 & .55 & .00 & -.13 & .00 & -.03 & .00 & -.02 & .00 & .25 & .00 \\
\hline & Leaderlike & & Follower & 4 & .40 & .11 & -.17 & .09 & .28 & .19 & .32 & .09 & .17 & .20 \\
\hline & Modern & & Old-fashioned, traditional & 6 & .40 & .11 & .01 & .21 & -.02 & .29 & .08 & .17 & .36 & .17 \\
\hline & Passionate & & Unfeeling & 3 & .46 & .03 & .30 & .04 & -.01 & .09 & -.14 & .00 & .37 & .08 \\
\hline & Progressive & & Preserving & 4 & .44 & .20 & -.06 & .08 & .07 & .19 & .08 & .10 & .32 & .16 \\
\hline & Spontaneous & & Inhibited & 5 & .59 & .11 & .09 & .07 & -.17 & .21 & .23 & .05 & .22 & .17 \\
\hline & & & & Factor I & & & & & & & & & & \\
\hline \multirow[t]{2}{*}{$\mathrm{II}+\mathrm{I}+$} & Generous & $\mathrm{II}-\mathrm{I}-$ & Stingy & 7 & .26 & .10 & .52 & .07 & .02 & .11 & .02 & .04 & .09 & .14 \\
\hline & Warm & & Cold & 11 & .35 & .09 & .60 & .06 & .10 & .07 & .05 & .10 & .07 & .13 \\
\hline \multirow[t]{2}{*}{$\mathrm{II}+\mathrm{I}-$} & Diplomatic & $\mathrm{II}-\mathrm{I}+$ & Outspoken & 4 & -.33 & .14 & .36 & .16 & .17 & .08 & -.11 & .13 & .10 & .17 \\
\hline & Humble & & Proud & 5 & -.16 & .36 & .25 & .35 & .00 & .11 & .00 & .06 & -.09 & .17 \\
\hline
\end{tabular}




\begin{tabular}{|c|c|c|c|c|c|c|c|c|c|c|c|c|c|c|}
\hline \multirow[b]{3}{*}{ Facet } & \multirow[b]{3}{*}{ Term } & \multirow[b]{3}{*}{ Facet } & \multirow[b]{3}{*}{ Anchors } & \multirow{3}{*}{$\begin{array}{l}N \text { of } \\
\text { data } \\
\text { sets }\end{array}$} & \multicolumn{10}{|c|}{ Faetor loadings across data sets ${ }^{a}$} \\
\hline & & & & & \multicolumn{2}{|c|}{ Factor I } & \multicolumn{2}{|c|}{ Factor II } & \multicolumn{2}{|c|}{ Factor 1II } & \multicolumn{2}{|c|}{ Factor IV } & \multicolumn{2}{|c|}{ Factor V } \\
\hline & & & & & $M$ & $S D$ & $M$ & $S D$ & $M$ & $S D$ & $M$ & $S D$ & $M$ & $S D$ \\
\hline \multirow[t]{3}{*}{$\mathbf{I I}+\mathbf{I I}+$} & Acquiescent & II-II-- & Antagonistic & 3 & .04 & .09 & .60 & .07 & .06 & .09 & .10 & .10 & -.10 & .06 \\
\hline & Mild, gentle & & I. Ieadst rong & 4 & -.07 & .25 & .80 & .01 & .07 & .17 & .07 & .12 & .14 & .04 \\
\hline & Softhearted & & Ruthiess & 3 & .11 & .14 & .72 & .03 & .12 & .07 & -.12 & .02 & .12 & .11 \\
\hline \multirow{8}{*}{$\mathrm{II}+\mathrm{III}+$} & Courteous & $11-111-$ & Rude & 3 & .03 & .13 & .59 & .05 & .33 & .07 & .05 & .07 & .20 & .10 \\
\hline & Empatinic & & Self-centered & 4 & .05 & .15 & .54 & .14 & .18 & .17 & .01 & .07 & -.05 & .17 \\
\hline & Fait & & Unfair & 7 & .07 & .07 & .52 & .08 & .37 & .04 & .17 & .08 & .22 & .11 \\
\hline & lelpful & & Uncooperative & 3 & .28 & .04 & .55 & .10 & .35 & .10 & .04 & .04 & .17 & .04 \\
\hline & Kind & & Unkind & 2 & .06 & .00 & .55 & .07 & .14 & .04 & .01 & .19 & .16 & .04 \\
\hline & polite & & Rude & 2 & .06 & .17 & .50 & .12 & .22 &.$I 1$ & .03 & .10 & .15 & .01 \\
\hline & Silless & & Selfish & 3 & -.03 & .01 & .55 & .08 & .14 & .09 & .05 & .03 & .01 & .05 \\
\hline & lacoul & & Blunt & 4 & -.08 & .07 & .48 & .26 & .18 & .06 & -.07 & .14 & .09 & .22 \\
\hline \multirow[t]{12}{*}{$\| 1+1 V+$} & Agrceable & $11 \mathrm{IV}$ & Critical or disagrecable & 11 & .06 & .08 & .48 & .20 & .08 & .17 & .10 & 30 & .09 & .16 \\
\hline & Cooperalive & & $\begin{array}{l}\text { Negativistie, stubborn, or } \\
\text { uncooperative }\end{array}$ & 10 & .20 & .20 & .54 & .21 & .09 &. $\mathrm{IO}$ & .21 & .12 & .15 & .17 \\
\hline & lexible & & Inflexible or stubborn & 7 & .22 & .13 & .44 & .09 & .00 & .07 & .24 & .08 & .22 & .14 \\
\hline & Forgiving & & Vengeful & 3 & .13 & .05 & .68 & .03 & .17 & .07 & .21 & .06 & .09 & .03 \\
\hline & Good-nalured & & Irritable & 12 & .05 & .18 & .59 & .21 & .07 & .08 & .25 & .23 & .13 & .19 \\
\hline & Nol jeillous & & Jealous & 4 & .02 & .22 & .53 & .18 & .09 & .11 & .42 & .10 & .05 & .06 \\
\hline & Nol énvious & & Envious/jealous & 7 & .07 & .10 & .34 & .23 & .23 & .11 & .34 & .11 & .09 & .16 \\
\hline & Sicidly & & Moody & 1 & .24 & .00 & .38 & .00 & .08 & .00 & .38 & .00 & -.22 & .00 \\
\hline & Tolerant & & Impaticnt & 4 & --.07 & .07 & .58 & .16 & .04 & .06 & .19 & .13 & .21 & .31 \\
\hline & Trusthul & & Distrustlul & 4 & .14 & 13 & .53 & .10 & .05 & .08 & .15 & .08 & -.14 & .14 \\
\hline & Irusting & & Suspicious & 3 & .19 & .04 & .60 & .02 & .04 & .07 & .17 & .03 & .04 & .10 \\
\hline & Unselfist & & Selinsh & 4 & -.02 & .16 & .51 & .08 & .08 & .12 & .13 & .13 & -.08 & .09 \\
\hline \multirow[t]{2}{*}{$11+\mathrm{V}+$} & Onen-minded & $\mathrm{II}-\mathrm{V}-$ & Narrow-minded & 3 & .23 & .08 & .46 & .07 & .18 & .05 & .20 & .09 & .46 & .06 \\
\hline & Sympathetic & & Callous & 3 & .24 & .06 & .59 & .07 & .22 & .03 & -.07 & .04 & .32 & .19 \\
\hline \multirow[t]{2}{*}{$\mathrm{Il}+\mathrm{V}-$} & Gullible & $11-V \cdot I$ & Cynical & 3 & .07 & .06 & .51 & .10 & $\cdots .07$ & .08 & -.11 & .07 & -.23 & .06 \\
\hline & 1.xnient & & Critical & 5 & -.06 & .11 & .56 & .06 & -.14 & .05 & .15 & .05 & -.18 & .12 \\
\hline \multicolumn{15}{|c|}{ Factor III } \\
\hline \multirow[t]{2}{*}{$111+1+$} & Ambitious & III I & Nimless or apathertic & 7 & .23 & .13 & .01 & .12 & 62 & .15 & .10 & .16 & .12 & .13 \\
\hline & Serious & & Frivolous & 2 & .04 & .33 & .11 & .11 & .54 & .06 & -.15 & .11 & .04 & .01 \\
\hline \multirow[t]{4}{*}{ III $+1-$} & Businesslike & $1111+4$ & Playlul & 3 & -.10 & .14 & .10 & .01 & .55 & .05 & .10 & .09 & -05 & .03 \\
\hline & Cautious & & Rash & 2 & -.29 & .03 & .13 & .15 & .49 & .00 & .10 & .13 & .11 & 01 \\
\hline & Deliberate & & Thoughtless & 3 & -.25 & .15 & .19 & .05 & .56 & .10 & .16 & .13 & .09 & .04 \\
\hline & Not impulse ridden & & Impulse ridden & 3 & -.27 & .03 & .18 & .02 & .53 & .13 & .23 & .06 & .00 & .20 \\
\hline \multirow[t]{4}{*}{$111+11+$} & Conscientious & III-11- & Negligent & 11 & .03 & .07 & .19 & .11 & .70 & .11 & -.02 & .13 & .10 & .15 \\
\hline & Reliable & & Undependable & 7 & .07 & .06 & .27 & .11 & .64 & .13 & .07 & .07 & .13 & .07 \\
\hline & Responsible & & lrresponsible or undependabte & 10 & .05 & .15 & .22 & .19 & .64 & .12 & .10 & .12 & .14 & .16 \\
\hline & Trustworthy & & Unreliable & 4 & -.01 & .06 & .24 & .21 & .56 & .05 & .10 & .06 & .06 & .20 \\
\hline $111+11-$ & Industrious & $1 I I-1 I-$ & Easy-going & 4 & .10 & .26 & --.06 & .37 & .42 & .22 & -.12 & .13 & .09 & .16 \\
\hline
\end{tabular}


Factor loadings across data sets $\mathrm{s}^{\mathrm{a}}$

\begin{tabular}{|c|c|c|c|c|c|c|c|c|c|c|c|c|c|c|}
\hline & \multirow[b]{3}{*}{ Term } & \multirow{3}{*}{ Facet } & \multirow[b]{3}{*}{ Anchors } & & & & & & & & & & & \\
\hline \multirow[b]{2}{*}{ Facet } & & & & \multirow{2}{*}{$\begin{array}{l}N \text { of } \\
\text { data } \\
\text { sets }\end{array}$} & \multicolumn{2}{|c|}{ Factor I } & \multicolumn{2}{|c|}{ Factor II } & \multicolumn{2}{|c|}{ Factor III } & \multicolumn{2}{|c|}{ Factor IV } & \multicolumn{2}{|c|}{ Factor V } \\
\hline & & & & & $M$ & $S D$ & $M$ & $S D$ & $M$ & $S D$ & $M$ & $S D$ & $M$ & $S D$ \\
\hline \multirow{8}{*}{ III + III + } & Careful & IIII-III- & Careless & 9 & -.01 & .07 & .16 & .08 & .70 & .10 & -.01 & .16 & .04 & .14 \\
\hline & Fussy, tidy & & Careless & 4 & -.24 & .17 & -.06 & .06 & .67 & .17 & -.18 & .17 & .12 & .20 \\
\hline & Hardworking & & Lazy & 7 & .16 & .06 & .16 & .10 & .64 & .15 & .00 & .17 & .04 & .09 \\
\hline & Neat & & Sloppy & 3 & -.04 & .05 & .16 & .05 & .67 & .09 & .00 & .04 & -.04 & .01 \\
\hline & Punctual & & Late & 3 & -.01 & .09 & .16 & .13 & .59 & .02 & -.01 & .06 & -.08 & .04 \\
\hline & Scrupulous & & Lax or unscrupulous & 7 & -.16 & .20 & .13 & .26 & .66 & .17 & -.02 & .05 & .10 & .10 \\
\hline & Thrifty & & Extravagant & 2 & -.18 & .07 & -.06 & .02 & .34 & .31 & .08 & .12 & -.07 & .11 \\
\hline & Well organized & & Disorganized & 3 & -.05 & .03 & .02 & .08 & .71 & .03 & .06 & .17 & .28 & .34 \\
\hline \multirow[t]{5}{*}{ III+IV+ } & Persevering & III-IV- & $\begin{array}{l}\text { Quitting or quitting, } \\
\text { fickle }\end{array}$ & 11 & .06 & .14 & .05 & .17 & .56 & .13 & .21 & .23 & .18 & .14 \\
\hline & Practical & & Impractical & 7 & .19 & .20 & .14 & .11 & .45 & .13 & .16 & .06 & .12 & .11 \\
\hline & Self-disciplined & & Weak willed & 3 & .03 & .05 & .02 & .06 & .65 & .04 & .28 & .02 & .20 & .06 \\
\hline & Stable & & Unstable & 4 & -.10 & .10 & .16 & .09 & .37 & .20 & .15 & .21 & .19 & .15 \\
\hline & Wcll-read & & Unlettered & 2 & .19 & .11 & .12 & .04 & .43 & .16 & .40 & .11 & -.05 & .04 \\
\hline \multirow[t]{4}{*}{ III + V+ } & Clever & III-V- & Naive & 4 & .18 & .15 & -.09 & .16 & .34 & .34 & .19 & .14 & .28 & .05 \\
\hline & Lcarned & & Unlearned & 4 & .00 & .09 & .00 & .14 & .49 & .27 & .17 & .09 & .22 & .18 \\
\hline & Organized & & Disorganized & 4 & .03 & .10 & .02 & .11 & .67 & .02 & .07 & .17 & .16 & .13 \\
\hline & Thorough & & Careless & 2 & -.04 & .24 & .10 & .25 & .58 & .11 & .06 & .04 & .19 & .16 \\
\hline \multirow[t]{2}{*}{$\mathrm{III}+\mathrm{V}-$} & Rule abiding & $\mathrm{II} 1-\mathrm{V}+$ & Rule avoiding & 4 & .01 & .17 & .15 & .12 & .44 & .17 & -.26 & .34 & -.14 & .16 \\
\hline & & & & Factor I & & & & & & & & & & \\
\hline \multirow[t]{5}{*}{$\mathrm{IV}+\mathrm{I}+$} & Confident & IV-I- & Worried & 4 & .16 & .14 & .02 & .09 & .09 & .03 & .58 & .12 & .00 & .12 \\
\hline & Guilt free & & Guilt ridden & 1 & .20 & .00 & -.01 & .00 & .10 & .00 & .68 & .00 & .03 & .00 \\
\hline & Healthy & & Frail & 4 & .16 & .07 & -.07 & .20 & .27 & .20 & .28 & .12 & .08 & .22 \\
\hline & Secure & & Insecure & 7 & .38 & .13 & .02 & .05 & .37 & .09 & .53 & .14 & .09 & .06 \\
\hline & Self-satisfied & & Self-pitying & 3 & .26 & .10 & .04 & .06 & .21 & .05 & .58 & .05 & .06 & .05 \\
\hline $\mathrm{IV}+\mathrm{I}-$ & Unemotional & $\mathrm{IV}-\mathrm{I}+$ & Emotional & 7 & -.33 & .19 & -.10 & .17 & .05 & .15 & .42 & .17 & -.19 & .11 \\
\hline \multirow[t]{7}{*}{$\mathrm{IV}+\mathrm{II}+$} & At ease & IV-II- & Nervous & 7 & .15 & .19 & .16 & .08 & -.01 & .07 & .69 & .09 & .06 & .05 \\
\hline & Composed & & Excitable or moody & 8 & .09 & .22 & .28 & .25 & .13 & .06 & .56 & .12 & .11 & .14 \\
\hline & Even tempered & & Temperamental & 6 & .02 & .03 & .32 & .15 & .24 & .02 & .61 & .10 & .00 & .10 \\
\hline & Not hypochondriacal & & Hypochondriacal & 4 & .19 & .07 & .18 & .12 & .01 & .05 & .63 & .02 & .03 & .09 \\
\hline & Patient & & Impatient & 3 & -.15 & .12 & .43 & .12 & .17 & .18 & .43 & .02 & .06 & .04 \\
\hline & Poised & & $\begin{array}{l}\text { Nervous or nervous, } \\
\text { tense }\end{array}$ & 8 & .07 & .07 & .26 & .22 & .02 & .10 & .65 & .05 & .07 & .08 \\
\hline & Relaxed & & High strung or tense & 11 & .11 & .11 & .24 & .09 & -.04 & .05 & .67 & .09 & .01 & .08 \\
\hline \multirow[t]{6}{*}{ IV +III+ } & Contented & IV-III- & Discontented & 2 & .11 & .25 & .31 & .01 & .27 & .14 & .42 & .07 & .10 & .01 \\
\hline & Emotionally stable & & Unstable & 5 & .11 & .04 & .12 & .09 & .36 & .06 & .63 & .04 & .07 & .05 \\
\hline & Forward looking & & Reminiscent & 4 & -.06 & .23 & .19 & .23 & .27 & .07 & .13 & .31 & .18 & .27 \\
\hline & Hardy & & Vulnerable & 3 & .13 & .02 & -.12 & .09 & .18 & .08 & .60 & .09 & -.07 & .17 \\
\hline & Objective & & Subjective & 5 & -.12 & .02 & .15 & .03 & .30 & .06 & .35 & .11 & .20 & .09 \\
\hline & Self-reliant & & Helpless & 3 & .28 & .08 & -.04 & .04 & .45 & .09 & .47 & .17 & .18 & .03 \\
\hline $\mathbf{I V}+\mathbf{I V}+$ & Calm & IV-IV- & $\begin{array}{l}\text { Angry, anxious, or } \\
\text { worrying }\end{array}$ & 13 & -.02 & .18 & .11 & .26 & .01 & .07 & .60 & .23 & .05 & .18 \\
\hline
\end{tabular}




\begin{tabular}{|c|c|c|c|c|c|c|c|c|c|c|c|c|c|c|}
\hline \multirow[b]{3}{*}{ Facet } & \multirow[b]{3}{*}{ Term } & \multirow[b]{3}{*}{ Facet } & \multirow[b]{3}{*}{ Anchors } & \multirow{3}{*}{$\begin{array}{l}N \text { of } \\
\text { data } \\
\text { sets }\end{array}$} & \multicolumn{10}{|c|}{ Factor loadings across data sets ${ }^{\mathrm{a}}$} \\
\hline & & & & & \multicolumn{2}{|c|}{ Factor I } & \multicolumn{2}{|c|}{ Factor II } & \multicolumn{2}{|c|}{ Factor III } & \multicolumn{2}{|c|}{ Factor IV } & \multicolumn{2}{|c|}{ Factor V } \\
\hline & & & & & $M$ & $S D$ & $M$ & $S D$ & $M$ & $S D$ & $M$ & $S D$ & $M$ & $S D$ \\
\hline & & & & actor & & & & & & & & & & \\
\hline \multirow{7}{*}{$\mathrm{V}+\mathrm{I}+$} & Experimenting & $\mathrm{V}-\mathrm{I}-$ & Routine & 4 & .46 & .16 & -.07 & .11 & .08 & .30 & .14 & .14 & .47 & .10 \\
\hline & Independent & & Conforming & 5 & .20 & .13 & -.23 & .07 & -.01 & .15 & .17 & .11 & .48 & .02 \\
\hline & Liberal & & Conservative & 9 & .16 & .13 & .11 & .16 & -.13 & .24 & -.02 & .13 & .49 & .15 \\
\hline & Original & & Conventional & 3 & .25 & .12 & .01 & .08 & -.10 & .06 & .05 & .05 & .61 & .06 \\
\hline & Prefer variety & & Prefer routine & 3 & .40 & .09 & -.01 & .13 & --.22 & .01 & .07 & .08 & .45 & .02 \\
\hline & Sophisticated & & Unsophisticated & 2 & .18 & .07 & -.07 & .13 & .01 & .04 & .10 & .08 & .58 & .21 \\
\hline & Untraditional & & Traditional & 3 & .20 & .19 & .06 & .10 & -.19 & .15 & .04 & .09 & .45 & .05 \\
\hline V+II+ & Reflective & V-II- & Unreflective & 2 & -.08 & .13 & .20 & .03 & -.02 & .03 & -.04 & .05 & .42 & .16 \\
\hline $\mathrm{V}+\mathrm{II}-$. & Complex & V-II+ & Simple & 9 & -.03 & .07 & -.18 & .15 & .08 & .18 & -.06 & .26 & .32 & .37 \\
\hline \multirow[t]{10}{*}{$\mathrm{V}+\mathrm{III}+$} & Analytical & V-III- & Unanalytical & 7 & -.11 & .10 & -.07 & .08 & .31 & .15 & .05 & .08 & .46 & .08 \\
\hline & Broad interests & & Narrow interests & 3 & .17 & .09 & .16 & .04 & .29 & .09 & .18 & .07 & .57 & .05 \\
\hline & Cultured & & Uncultured & 7 & .01 & .07 & .16 & .12 & .27 & .15 & .07 & .08 & .52 & .13 \\
\hline & Curious & & Uninquisitive or uncurious & 7 & .13 & .10 & .14 & .08 & .23 & .19 & .05 & .14 & .50 & .06 \\
\hline & Intellectual & & $\begin{array}{l}\text { Unreflective or unreflective, } \\
\text { narrow }\end{array}$ & 8 & -.02 & .06 & .07 & .06 & .39 & .23 & .16 & .11 & .52 & .29 \\
\hline & Intelligent & & Stupid or unintelligent & 7 & .06 & .04 & .06 & .14 & .27 & .12 & .10 & .07 & .58 & .14 \\
\hline & Knowledgeable & & Ignorant & 5 & .00 & .04 & .05 & .08 & .31 & .12 & .11 & .03 & .64 & .09 \\
\hline & Perceptive & & Imperceptive & 7 & .09 & .06 & .07 & .09 & .29 & .12 & .13 & .03 & .53 & .20 \\
\hline & Polished, refined & & Crude, boorish & 4 & .08 & .08 & .33 & .06 & .38 & .12 & .08 & .06 & .50 & .05 \\
\hline & Refined & & Unrefined & 2 & .03 & .22 & .04 & .29 & .16 & .10 & .15 & .26 & .41 & .24 \\
\hline \multirow[t]{2}{*}{$V+H I I-$} & Clangeable & V. III + . & Predictable & 4 & .01 & .14 & -.05 & .19 & -.04 & .28 & -.09 & .08 & .56 & .22 \\
\hline & Unorthodox & & Traditional & 4 & .03 & .11 & -.04 & .23 & -.18 & .32 & -.04 & .12 & .55 & .21 \\
\hline $\mathrm{V}+\mathrm{IV}+$ & Aesthetic & V-IV- & Inartistic & 4 & .00 & .03 & .34 & .21 & .16 & .21 & .23 & .24 & .21 & .35 \\
\hline \multirow[t]{3}{*}{$V+V$} & Artistic & 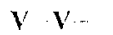 & Unartistic & 7 & -.01 & .10 & .09 & .06 & .08 & .20 & -.06 & .04 & .62 & .15 \\
\hline & Creative & & Ordinary or uncreative & 11 & .14 & .09 & .17 & $.1 \mathrm{I}$ & .15 & .22 & .09 & .19 & .47 & .30 \\
\hline & Inalginative & & $\begin{array}{l}\text { Down-to-earth or simple, direct, } \\
\text { or unimaginative }\end{array}$ & 13 & .11 & .09 & .14 & .19 & -.11 & .17 & .01 & .18 & .49 & .35 \\
\hline
\end{tabular}

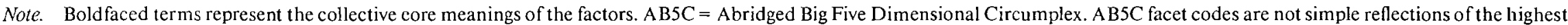
and second highest average loadings for reasons presented in footnote $I$ in the text. 


\section{Results}

\section{AB5C Depictions of Personality Terms}

Table 2 summarizes the final AB5C depictions for all personality terms. Of the 137 personality terms in this set, only 21 were factor pure. ${ }^{2}$ The remaining $85 \%$ were $\mathrm{AB} 5 \mathrm{C}$ factor blends. These factor blends represent the hypothesized culprits underlying disagreements about the FFM.

Positive emotions. Table 2 affirms the link between extraversion and positive emotions. The hearty, energetic positive emotions-affectionate, cheerful, enthusiastic, and friendlyreceived a I+II+ designation, whereas the milder positive emotions, generous and warm, were designated as II+I+. This supports McCrae and John's (1992; see also John, 1990a) suggestion that "Warmth, interpreted as heartiness and affection, is more closely related to [I] than [II]; interpreted as compassion and sympathy, it is more closely related to [II] than [I]" (p. 196). (Sympathetic was depicted as II+V+.)

Overlooked in previous discussions of positive emotions are blends other than I+II+ or II+I+. Proud (II-I+), for example, is positive from the perspective of the person experiencing this emotion but negative from the viewpoint of others. A significant cluster of II+IIIt terms-courteous, empathic, kind, and polite--describe positive emotions that also indicate mannerliness.

One might also note the significant number of terms that describe freedom from negative emotions (Tellegen, 1982) rather than the possession of positive emotions. These terms invariably involved IV+ but also involved II+ either as a primary (agreeable, good-natured, not jealous, not envious, trustful, and trusting) or secondary (at ease, composed, even tempered, patient, poised, and relaxed) part of the depiction. This underscores the fact that persons find others who express negative emotions to be more unpleasant and disagreeable than persons who do not express negative emotions. Other terms indicating freedom from negative affect included the I+IV+ comfortable and not lonely and the IV+I+ guilt free, secure, and self-satisfied.

Conformity. From the set of terms denoting conformity, only one (acquiescent, II+IIt) appeared to belong to the Agreeableness domain. The remainder were combinations of $\mathrm{III}+, \mathrm{V}-$, and $\mathrm{I}-$. These were rule abiding $(\mathrm{III}+\mathrm{V}-$ ) and traditional (vs. unorthodox; V-IIIt); conventional, conforming, and traditional (vs. experimenting and independent and untraditional; all $\mathrm{V}-\mathrm{I}-$ ); unassertive (vs. assertive; $\mathrm{I}-\mathrm{IV}-$ ) and submissive; and follower (vs. forceful and leaderlike; both $\mathrm{I}-\mathrm{V}-$ ).

Ambition. The term ambitious itself received a III+I+ designation, confirming the suggested double meaning given by McCrae and John (1992). The following terms, related primarily to "getting ahead (of others)," were included in the domain of Factor I: leaderlike and forceful (both $\mathrm{I}+\mathrm{V}+$ ), assertive (I+IV+), and dominant ( $\mathrm{I}+\mathrm{II}-$ ). Two terms indicating "getting things done" were more highly related to Factor III: industrious (III+II-) and hardworking (III+III+).

Impulsivity. Terms indicating impulsivity almost invariably involved the low end of Factor III. Careless (vs. careful or fussy, tidy) was the only pure III-III- term. In support of Eysenck's (Eysenck \& Eysenck, 1977) original placement of impulsivity with Extraversion, the following terms were designated III-I+ : thoughtless (vs. deliberate), impulse ridden (vs. not impulse ridden), and rash or careless (vs. cautious). This III-I+ designation seems to describe an extraverted type of impulsivity that might be related to sensation seeking (Birenbaum \& Montag, 1986; Zuckerman, Kuhlman, \& Camac, 1988). Eysenck's transfer of impulsivity to Psychoticism (II-III-) was supported by two terms with that designation: rude (vs. polite or courteous) and blunt (vs. tactful). A third term related to interpersonal impulsivity seems to be outspoken (vs. diplomatic, II-I+). Only one term, quitting (vs. persevering, III-IV-), fell into the domain of Neuroticism. Finally, two terms suggested a link between more desirable forms of impulsivity and Factor V: changeable (V+III-) and spontaneous (I+V+).

Intellect. Almost all intellect-related terms proved to be blends of Factors V and III, which explains why McCrae and Costa (1985b) located these terms with III, whereas Goldberg (1993) and Hogan and Johnson (1981) located them with V. However, only two terms (clever and learned) were III+V+. The remaining blends were V+III+: analytical. broad interests, cultured, curious, intellectual, intelligent, knowledgeable, perceptive, polished, and refined. In addition, two intellect-related terms were pure $\mathrm{V}+\mathrm{V}+$ : creative and imaginative.

\section{AB5C Depictions of Personality Scales}

Table 3 indicates the $\mathrm{AB} 5 \mathrm{C}$ structure of the scales proffered by the four research groups for assessing the FFM. Most of these scales are heterogeneous factor blends. Moreover, the blends differ across the four research groups. These differences point to differences in the conceptions of the various measures.

Factor I scales. Three of the four scales intended to assess Factor I received pure I+I+ designations. (Hogan \& Johnson's [1981] Power/Ambition scale was never intended to be a measure of pure extraversion; it showed a I+III+ character, consistent with McCrae and John's [1992] discussion of ambition.) Only McCrae and Costa's (1985b) scale was an AB5C blend: $\mathrm{I}+\mathrm{II}+$. This seems to be due to their view that positive emotions and warmth belong to Factor I. but, when plotted, these facets occupy an intermediate position between Factors I and II (McCrae \& Costa, 1989b).

Factor II scales. Three of the four Factor II scales received a II + IV + designation. This suggests two points. First, what these researchers mean by "agreeable" is possessing a pleasant disposition rather than conforming to others' wishes (otherwise, the scales would have been II+III-). Second, the II + IV+ character of these scales indicates that part of what makes persons likable is that they do not express negative affect (IV-). In Hogan's (1983) words, "Some persons use charm, tact, and understanding to achieve their interpersonal goals: trait neurotics use headaches, colds, and dysphoria to achieve theirs" (p. 80). Only McCrae and Costa's (1985b) Factor II scale received a II+I+ designation; this probably reflects their view: described earlier. on the location of warmth and positive emotions.

\footnotetext{
${ }^{2}$ One should note that our definition of factor pureness arbitrarily follows Hofstee et al.s (1992) convention (i.e., being located within $15^{\circ}$ of one of the primary factor axes). Dividing the circumplex into octants (Wiggins, 1979) rather than duodecants would broaden the area about the primary factor axes, increasing the number of factor-pure terms.
} 
Table 3

AB5C Structure of Big Five Scales From Different Research Groups

\begin{tabular}{|c|c|c|}
\hline Researchers & Scale name & AB5C designation \\
\hline \multicolumn{3}{|l|}{ Factor I } \\
\hline Goldberg (1992) & Surgency & $\mathrm{I}+\mathrm{I}+$ \\
\hline \multirow{2}{*}{ Hogan and Johnson $(1981)^{\mathrm{a}}$} & Sociality & $\mathrm{I}+\mathrm{I}+$ \\
\hline & Power & $\mathrm{I}+\mathrm{III}+$ \\
\hline McCrae and Costa (1985b) & Extraversion & $\mathrm{I}+\mathrm{II}+$ \\
\hline Norman (1963) & Extraversion/surgency & $\mathrm{I}+\mathrm{I}+$ \\
\hline \multicolumn{3}{|l|}{ Factor II } \\
\hline Goldberg (1992) & Agreeableness & $\mathrm{II}+\mathrm{IV}+$ \\
\hline Hogan and Johnson (1981) & Likeableness & II + IV+ \\
\hline McCrae and Costa (1985b) & Agreeableness & $\mathrm{II}+\mathrm{I}+$ \\
\hline Norman (1963) & Agreeableness & $\mathrm{II}+\mathrm{IV}+$ \\
\hline \multicolumn{3}{|l|}{ Factor III } \\
\hline Goldberg (1992) & Conscientiousness & III+III+ \\
\hline Hogan and Johnson (1981) & Conventionality & $\mathrm{III}+\mathrm{II}+$ \\
\hline McCrae and Costa (1985b) & Conscientiousness & $\mathrm{III}+\mathrm{V}+$ \\
\hline Norman (1963) & Conscientiousness & $\mathrm{III}+\mathrm{III}+$ \\
\hline \multicolumn{3}{|l|}{ Factor IV } \\
\hline Goldberg (1992) & Emotional Stability & $\mathrm{IV}+\mathrm{II}+$ \\
\hline Hogan and Johnson (1981) & Poise & $\mathrm{IV}+\mathrm{I}+$ \\
\hline McCrae and Costa (1985b) & Neuroticism (inflected) & $\mathrm{IV}+\mathrm{II}+$ \\
\hline Norman (1963) & Emotional stability & IV $+1 \mathrm{II}+$ \\
\hline \multicolumn{3}{|l|}{ Factor $\mathrm{V}$} \\
\hline Goldberg (1992) & Intellect & $\mathrm{V}+\mathrm{III}+$ \\
\hline \multirow[t]{2}{*}{ Hogan and Johnson (1981) } & Mentality & $\mathrm{V}+\mathrm{III}+$ \\
\hline & Novelty & $\mathrm{V}+\mathrm{I}+$ \\
\hline McCrae and Costa (1985b) & Openness to experience & $\mathrm{V}+\mathrm{I}+$ \\
\hline Norman (1963) & Culture & $\mathrm{V}+\mathrm{IlI}+$ \\
\hline
\end{tabular}

Note. $\mathrm{AB} 5 \mathrm{C}=$ Abridged Big Five Dimensional Circumplex.

${ }^{a}$ Revised scale names (Johnson, in press) used instead of original scale names.

Factor III scales. Norman's (1963) and Goldberg's (1992) Factor III scales proved to be purely III +III+. Hogan and Johnson's (1981) scale received a III+II+ designation, whereas McCrae and Costa’s (1985b) scale received a III+V+ designation. The III+II+ terms reliable, responsible, and trustworthy indicate that Hogan viewed Factor III in terms of interpersonal maturity and socialization. The III $+\mathrm{V}+$ terms clever, thorough, and organized indicate that $\mathrm{McCrae}$ and Costa leaned more toward Digman and Takemoto-Chock's (1981) interpretation of Factor III as organized directedness-purposefulness (vs. chaotic aimlessness) or will to achieve.

Factor IV scales. Goldberg's (1992), McCrae and Costa's (1985b), and Norman's (1963) Factor IV scales again showed the connection between emotional stability and agreeableness; all were IV+II+. Hogan and Johnson's (1981) IV+I+ Factor IV scale indicates their socioanalytic view (Hogan, 1983; Hogan \& Johnson, 1979; Hogan et al., 1978) that the most important affects are interpersonal rather than private (see John, 1990a; Saucier, 1992a; Wiggins \& Pincus, 1989; and particularly Lanning \& Gough's, 1991, comments on the link between extraversion and neuroticism in normal populations).

Factor $V$ scales. Three of the scales designed specifically to measure Factor $V$ were found to be V+III+ ; McCrae and Costa's (1985b) Factor V scale received a V+It designation. The V+III+ terms intellectual and intelligent (among others) showed that three of the four research groups favored an intellect interpretation of this factor. The $\mathrm{V}+\mathrm{I}+$ terms experimenting, original, prefers variety, and untraditional (among others) clearly under- score McCrae and Costa's openness-to-experience interpretation. Hogan and Johnson's (1981) version of an openness scale, Novelty, also received a V+I+ designation. Hogan and Johnson previously had assumed that the essence of openness was intellectual nonconformity, and therefore they expected their openness scale to be a V+III- blend (Hogan \& Johnson, 1979; Johnson, 1983). Their scale in fact contains two V+III- terms (unorthodox and changeable), but overall their scale suggested that openness to experience is a V+I+ blend.

\section{Discussion}

\section{Location of Specific Personality Terms Within the FFM}

As predicted, when personality terms are included by different research groups in different factors, these terms seem to be best described as $\mathrm{AB} 5 \mathrm{C}$ factor blends. Positive emotions are I+II+ or II+III+ blends; terms related to conformity are combinations of III+, I-, and $\mathrm{V}-$; ambition-related terms are III+I+ blends; intellectual terms are V+III+ blends; and terms related to impulsivity are blends of III-, II-, I+, and V+. That disagreements about the assignment of blends should arise is understandable; terms such as warm (II+I+) in a sense belong to both Factor II and Factor I.

Attempting to locate specific personality terms on one and only one of the broad five factors will always be problematic for terms that are blends. A parallel problem exists for inventories for which the primary scales are divided into smaller facets (e.g., 
Costa \& McCrae, 1985, 1992; Hogan \& Hogan, 1992). Simply dividing primary scales into facets assumes that the facets represent factor-pure elements or at least that any secondary aspect to the facet is unimportant. Yet it may be a mistake to limit a facet's scoring to only one scale if that facet scale represents a factor blend rather than a factor-pure element (Saucier, 1992a). As an alternative to locking the smaller facet scales into the scoring of one primary scale, researchers might experiment with several scoring schemes in which facets with similar AB5C codes are brought together. Hogan (1986) seemed to have used this idea when he recombined his facet scales to predict different kinds of occupational performance.

Because blends are associated with more than one factor, the following question arises: Do disagreements across research programs on the location of personality terms merely reflect differences in theoretical preference, taste, and opinion, or is it possible that one view is more "correct" than another? One might argue that researchers are making mistakes when they assign terms, a priori, to factors on which the terms do not show their primary loadings-both in their own data sets and in others. These mislocations seem to be driven by the researchers' theoretical preconceptions.

For example, Angleitner and Ostendorf (1991) pointed to several specific terms mislocated by McCrae and Costa (1985b). Their a priori assignment of impulse ridden (III-I+) to IV- was seemingly motivated by the fact that the Neuroticism scale from their NEO inventory contains an Impulsivity facet. Similarly, they placed warm $(\mathrm{II}+\mathrm{I}+)$ under I+ because Extraversion in their NEO model contains a Warmth facet. Emotionally stable (IV+III), fair (II+III+), knowledgeable, intelligent, perceptive, and cultured (all V+III+) were all mislocated on the secondary dimension of the AB5C code, III+. Attempting to place these last four traits on Factor III instead of Factor $V$ appears to have been a way of denying an intellect interpretation of Factor $\mathrm{V}$. Lest the reader think we are picking on McCrae and Costa (1985b), Hogan and Johnson (1981) mislocated the positive emotions cheerful (I+II+) and self-assured (I+IV+) on Factor IV.

\section{The Nature of the Big Five From an AB5C Perspective}

The present analyses also indicate that when two researchers hold different interpretations of a factor from the FFM, they use scales designated by divergent $\mathrm{AB} 5 \mathrm{C}$ codes. McCrae and Costa's (1985b) view that positive emotions and warmth belong to Factor I caused their Factor I and II scales to take on a I+II+ and $\mathrm{I}+\mathrm{I}+$ character, respectively; this differs from the other researchers whose Factor I scales were $\mathrm{I}+\mathrm{I}+$ and Factor II scales were II +IV+. McCrae and Costa's Factor III scale received a $\mathrm{III}+\mathrm{V}+$ designation, reflecting their view that intellect belongs to this factor rather than to the fifth factor. This view is consistent with the interpretation of Factor III as organized purposefulness or intellectual achievement. In contrast, Hogan and Johnson's (1981) scale was III+Il+, reflecting a view of Factor III as interpersonal maturity and impulse control. Finally, McCrae and Costa's (1985b) Factor V scale, which they said measures openness to experience, received a $\mathrm{V}+\mathrm{I}+$ designation. The other researchers, who favored an intellect interpretation of Factor V, used V+III+ scales.

Again, an important question is whether alternative interpre- tations of the factors are equally valid or whether certain interpretations are, in some way, more correct or optimal (Goldberg, 1993). Some would regard this question as intractable, because interpretation of factors depends on rotation, and rotational decisions are arbitrary (see McCrae \& Costa, 1989b, p. 591). A rotation of axes can cause terms that were $\mathrm{II}+\mathrm{HII}+$ in the original solution to become II+II+ or III+III+ (depending on the direction of rotation), and this will effectively change the nature of Factors II and III.

Yet the rotation problem does not keep researchers from thinking about the possibility of "identifying the 'correct' axes in a plane" (McCrae \& Costa, 1989b, p. 592). We do not claim to be able to offer the correct interpretation of factors, but we have a suggestion for a method of interpreting the Big Five that transcends to some degree the preconceptions of different researchers. We suggest that one can look at the factor-pure terms (I+I+, II+II+, etc.) in our study, for these terms might be conceptualized (in a metaphorical sense) as the "elemental essence" of the factor. ${ }^{3}$ Metaphorically, a set of factor-pure terms represents a single-element compound such as $\mathrm{O}_{2}$, whereas the nonpure terms represent two-element compounds such as $\mathrm{H}_{2} \mathrm{O}$ (see Hogan. 1983; Hogan \& Johnson, 1981). Although factor-pure terms are not elemental in the fixed, absolute fashion of chemical elements in the periodic table, they do represent a standard on which a number of research programs converge.

Like Hofstee et al., 1992, we found relatively few factor-pure as opposed to factor-blend terms; thus, these pure terms might not give us a complete picture of the core of each factor (see also the comments in Footnote 2). On the basis of the available data, however, one might draw the following tentative conclusions about the elemental essences or core meanings of each factor. The pure $\mathrm{I}+\mathrm{I}+$ items extraverted, frank/open, fun loving, sociable, talkative, and straightforward suggest that the core of Factor I is social expressiveness or communicativeness.

\footnotetext{
${ }^{3}$ In using the term elemental essence, it should be clear that we are speaking metaphorically and not metaphysically. There is no assumption here that factors in a factor analysis are fundamental entities with intrinsic qualities or powers to produce effects. This is where the chemical analogy breaks down, for at a descriptive level chemical elements are perceived to have intrinsic qualities and the power to produce effects (Weimer, 1984, p. 163). Factors, in contrast, are of course simply arbitrary mathematical abstractions for summarizing numerical data and have no more absolute metaphysical reality than does a mathematical line constructed by the least squares method over a scatterplot of points. Orthogonal factors seem to be similar to what Rosch and Mervis (1975) called natural categories, which "tend to become organized in such a way that they reflect the correlational structure of the environment in a manner which renders them maximally discriminable from each other" (p. 575). Alternatively, a factor can be understood as a Weberian ideal type (Gerth \& Mills, 1946), which is a "logically precise conception" that is "removed from historical reality" (pp. 59-60) in the sense that a pure, mathematically precise line $(y=m x+b)$ is removed from the lines one draws in "reality" (i.e., the physical world; Hogan, 1983, p. 61). Both the Roschian prototype and Weberian ideal type conceptions in a sense derive from a sort of Platonic idealism in which the mind uses idealized fictions to comprehend clearly fuzzy things in the physical world (Weimer, 1973). It remains to be seen how a personological taxonomy derived from the formalism of factor analysis will compare with yet-to-be-developed taxonomies on the basis of the physical substrates (genes and neurophysiology) of personality.
} 
The II+II+ cell contains acquiescent, mild/gentle, and softhearted. Because past research has indicated that Factor II is highly desirable, perhaps these terms suggest the type of femininity valued by Taoists (Waley, 1958): virtue through yielding, pliancy, softness, suppleness, and receptivity. "A man is born gentle and weak. At his death he is hard and stiff. Green plants are tender and filled with sap. At their death they are withered and dry. Therefore the stiff and unbending is the disciple of death. The gentle and yielding is the disciple of life. Thus an army without flexibility never wins a battle. A tree that is unbending is easily broken. The hard and strong will fall. The soft and weak will overcome" (Feng \& English, 1972, chap. 76). The link between femininity and Factor II has been noted previously by several researchers (John, 1990a; McCrae et al., 1993).

Factor III was defined by the following pure items: carefiul, fussy/tidy, hardworking, neat, punctual, scrupulous, thrifty, and well organized. Of all these terms, only one-hardworkingpoints to a directedness-purposefulness-achievement interpretation of Factor III. The other terms describe an abstemious, exacting, orderly, prudent, restrained, temperate person, a person who avoids excesses and pays close attention to detail. Perhaps Tellegen's (1982) label constraint fits the III+III+ terms most aptly.

Factor IV, a relatively noncontroversial factor, was defined by one pure IV+IV+ item, calm. This suggests that the core of IV is freedom from negative affect (Tellegen, 1985).

For Factor $\mathrm{V}$, we found three $\mathrm{V}+\mathrm{V}+$ items, artistic, creative, and imaginative. This suggests that creativity is not merely related to Factor V (cf. McCrae, 1987) but rather lies at the core of the meaning of Factor V. A creativity interpretation of Factor $\mathrm{V}$ has been suggested also by Saucier (1992b).

To identify the elemental essence or core meaning of each factor in terms of pure-factor items is not to claim that the pure items exhaustively and fully define each factor. Rather, each of the five dimensions should be seen as categories for which the core meanings are defined by pure items that function as prototypical exemplars (Rosch, 1977; Rosch \& Mervis, 1975). The nonpure items are less prototypical but are still important for defining the overall meaning of each factor. Yet these nonpure items are most clearly understood by reference to the pure, core meanings of the two factors that define them.

What are we to make of the fact that (with the exception of Extraversion), most of the scales used by FFM researchers are defined by blends rather than factor-pure terms? We suggest this may be due to what Hofstee et al. (1992) called the natural "promiscuity" of factors, that certain factors cannot help but couple with each other, producing many hybrid offspring. We find this amusing biological metaphor consistent with our chemical metaphor. Factors (defined by pure terms) are like chemical elements, and the blends defined by factors within a circumplex are like chemical compounds. Chemical compounds represent elements that couple naturally rather than randomly. (Hofstee et al.s, 1992, original, nondynamic, metaphor was astronomical -in the sky one sees clusters of stars in some areas and blank space in others.)

Whatever the source of the factors' natural coupling tendency, it is a fact that certain blends tend to be overrepresented, both in Hofstee et al's (1992) analysis and in the current one. Nineteen terms were II+IV+ or IV+II+, which explains why six of eight Factor II and IV scales were blends of the two factors (see Peabody \& Goldberg, 1989, for a protracted discussion on the relationship between Factors II and IV). The basis of the promiscuity of Factor II with Factor IV was suggested earlier, mainly that neurotic people are unpleasant to be around (see also Saucier, 1992a).

Another notable coupling can be found between Factors III and V (14 terms). The promiscuity here was responsible for three intellect-based Factor V scales and one intellect-based Factor III scale. Factor V also coupled often with I (14 terms), forming the basis for McCrae and Costa's (1985b) opennessbased Factor V scale (see Peabody \& Goldberg's, 1989, discussion of controlled vs. expressive intellect). Factor I also appeared to be attracted to Factor IV (12 terms); this coupling made possible Hogan and Johnson's (1981) social poise Factor IV scale (see Lanning \& Gough, 1991; Saucier, 1992a).

Finally, positive emotions seem to be the basis for I+II+ couplings ( 7 terms and two scales). Factors I and III coupled to form ambitious and energetic traits ( 7 terms and one scale). In addition, couplings between Factors II and III (12 terms and one scale) described personality traits related to moral conduct (Hogan et al., 1978).

\section{Are Nonpure FFM Scales a Problem?}

Because FFM scales typically contain more blend terms than pure terms, these scales will inevitably intercorrelate, despite the fact that the FFM derives from a varimax-rotated, orthogonal factor-analytic solution: This can be a source of annoyance for persons attempting to use the FFM scales in the prediction of a criterion through multiple regression. Five completely independent scales will certainly be more useful in this context than intercorrelated scales. Yet de Raad, Hendricks, and Hofstee (1991) doubted that pure scales could be created by removing blend terms. They argued that refactoring the correlation matrix after blend terms are removed will produce new blend terms. This proposition can be investigated empirically, but a simple alternative method for deriving orthogonal predictors is to use factor scores (Goldberg, 1990, 1992; McCrae \& Costa, 1989a).

On the other hand, the intercorrelations among factor-derived scales justifies the summation of facets into an overall scale score. Take, for example, the four factors of Hogan's (1969) Empathy scale identified by Johnson, Cheek, and Smither (1983): Social Self-Confidence (I+rVH), Even-temperedness (II+IV+), Sensitivity (II+V+), and Nonconformity (V+I+). Empathy subscales corresponding to these four factors showedlike McCrae and Costa's (1985a) scales - a convergent-discriminant pattern of relations with other, external variables. This has led some researchers (e.g., Edelmann \& McCusker, 1986) to question whether the Empathy scale measures empathy at all or rather four separate unrelated factors. The low homogeneity of the scale has also been cited to make the same argument (Cross \& Sharpley, 1982). From an AB5C perspective, however, it is perfectly reasonable for a scale to consist of distinct parts (because the $A B 5 C$ codes of the parts are not identical) and yet "hang together" as a coherent whole on the basis of "family resemblance" (Rosch \& Mervis, 1975), stemming from a partial overlap in the AB5C codes. 


\section{Applicability of the Present Analyses to FFM Questionnaires}

Implicit in the above discussion of Hogan's (1969) Empathy scale and in previous references to other personality inventories is the assumption that questionnaire scales as well as single personality terms can be described in AB5C terminology. Justification for this position can be found in the research of Johnson (1992), who demonstrated that AB5C circumplexes generated by single terms, by scales of the California Psychological Inventory (Gough, 1987; Johnson, 1991), and by scales of the Hogan Personality Inventory are simply rotational variants of each other.

The construction and validation of omnibus personality inventories is a laborious, time-consuming process, so it is not surprising when the author of an inventory claims to have constructed the best (if not the "one true") measure of a construct (see Wicklund, 1990a, 1990b). Pride of ownership may lead researchers to defend vigorously the distinctiveness of their measure. Consider, for example, McCrae and Costas (1985a) painstaking attempt to distinguish Openness (their version of Factor V) from Extraversion (I+), low Conscientiousness (III-), and Hogan's (1986) version of Factor V, Intellectance. Although they present cogent conceptual arguments for the distinctiveness of the Openness construct, McCrae and Costa nevertheless readily admitted that there is overlap between their Openness scale and measures of these other constructs. They noted that their Openness scale correlates 28 with Extraversion (Factor I). In an unpublished study, Miller, Thayer, and Johnson (1990) found that McCrae and Costa's adjectival measure of openness correlated .45 with Hogan's (1986) Intellectance (V) scale and -.55 with Hogan's Prudence (III) scale.

The $\mathrm{AB} 5 \mathrm{C}$ model readily explains overlap between scales that purportedly assess different constructs. If Costa and McCrae's (1985) Openness scale, like their adjectival scale, carries a $\mathrm{V}+\mathrm{I}+$ designation, the correlation with Extraversion (I+) is virtually guaranteed. Similarly, a correlation with Intellectance $(\mathrm{V}+\mathrm{III}+)$ is expected. Finally, to the degree that low scores on a Factor III scale reflect aspects of nonconformity, such as rule avoiding (III-V+), changeable ( $\mathrm{V}+\mathrm{III}-)$, and unorthodox $(\mathrm{V}+\mathrm{III}-$, the scale can be expected to overlap with an openness scale that also contains aspects of nonconformity, such as original ( $\mathrm{V}+\mathrm{I}+)$ and untraditional $(\mathrm{V}+\mathrm{I}+)$.

McCrae and John (1992) stressed that the usefulness of the FFM is seriously compromised when researchers misinterpret or mislabel factors from their own or others' research. They suggest, for example, that Hogan (1983) was simply wrong to associate McCrae and Costas (1985a) Openness to Experience construct with low Conscientiousness (III-). We would argue that such "misinterpretations" are often cases in which the two scales are $A B 5 C$ biends that overlap on only one of the two aspects of the blend.

Goldberg (1992) has stressed the need for a set of relatively pure markers for the Big Five. Given the inherent promiscuity of personality terms, we are uncertain whether the development of pure markers is possible. We have shown, however, that scales proposed by different researchers to assess the FFM often tend to be blends rather than pure markers of these factors. We propose as a tentative conceptual standard for each factor the intersection of the work of Goldberg (1990), Hogan and Johnson (1981), McCrae and Costa (1985b), and Norman (1963). Future research can assess whether the inclusion of measures offered by other researchers would substantially change the present findings. Through the methodology of the $\mathrm{AB} 5 \mathrm{C}$, we found a commonality in the way these different researchers conceptualize and measure the Big Five. This provides us with a consensual definition of the core of each factor: Factor I, Social Communicativeness; Factor II, Softness; Factor III, Constraint; Factor IV, Freedom From Negative Affect; and Factor V, Creativity.

\section{References}

Angleitner, A., \& Ostendorf, F. (1991, June). Temperament and the Big Five factors of personality: Paper presented at the conference on the Developing Structure of Temperament and Personality in Childhood, Netherlands Institute for Advanced Study in the Humanities and Social Sciences, Wassenaar, The Netheriands.

Birenbaum, M., \& Montag, I. (1986). On the location of the sensation seeking construct in the personality domain. Multivariate Behavioral Research, 21, 357-373.

Costa, P. T., Jr.. \& McCrae, R. R. (1985). The NEO Personality Inventory manual. Odessa, FL: Psychological Assessment Resources.

Costa, P. T., Jr., \& McCrae, R. R. (1992). Revised NEO Personality Inventory (NEO PI-RTM) and NEO Five-Factor Inventory (NEOFFI) professional manual. Odessa, FL: Psychological Assessment Resources.

Costa, P. T., Jr., McCrae, R. R., \& Dye, D. A. (1991). Facet scales for Agreeableness and Conscientiousness: A revision of the NEO Personality Inventory. Personality and Individual Differences, 12, 887898.

Cross, D. G., \& Sharpley, C. F. (1982). Measurement of empathy with the Hogan Empathy Scale. Psychological Reports, 50, 62.

de Raad, B., Hendricks, J., \& Hofstee, W. K. B. (1991, January). Toward a refined structure of personality. Colloquium presentation to the Department of Psychology, Universität Bielefeld, Bielefeld, Germany.

Digman, J. M. (1990). Personality structure: Emergence of the five-factor model. Annual Review of Psichology, 41, 417-440.

Digman, J. M., \& Takemoto-Chock, N. K. (1981). Factors in the natural language of personality: Re-analysis, comparison, and interpretation of six major studies. Multivariate Behavioral Research, 16, $149-170$

Edelmann, R. J.. \& McCusker, G. (1986). Introversion, neuroticism, empathy, and embarrassibility. Personality and Individual Differences, 7, 133-140

Eysenck, S. B. G.. \& Eysenck, H. J. (1977). The place of impulsiveness in a dimensional system of personality description. British Joumal of Social and Clinical Psychology, 16, 7-68.

Feng, G.-F., \& English, J. (Trans.). (1972). Tao Te Ching. New York: Vintage Books.

Gerth, H. H., \& Mills, C. W. (1946), From Max Weber: Essays in sociology. New York: Oxford University Press.

Goldberg, L. R. (1980. May). Some ruminations about the structure of individual differences: Developing a common lexicon for the major characteristics of human personality. Paper presented at the Western Psychological Association Convention, Honolulu, HI.

Goldberg, L. R. (1983, June). The magical number five, phus or minus two: Some conjectures on the dimensionality of personality descrip- 
tions. Paper presented at a research seminar, Gerontology Research Center, Baltimore.

Goldberg, L. R. (1990). An alternative "description of personality": The Big-Five factor structure. Journal of Personality and Social Psychology, 59, 1216-1229.

Goldberg, L. R. (1992). The development of markers for the Big-Five factor structure. Psychological Assessment, 4, 26-42.

Goldberg, L. R. (1993). The structure of phenotypic personality traits. American Psychologist, 48, 26-34.

Gough, H. G. (1987). California Psychological Inventory administrator's guide. Palo Alto, CA: Consulting Psychologists Press.

Guilford, J. P. (1975). Factors and factors of personality. Psychological Bulletin, 82, 802-814.

Hofstee, W. K. B., de Raad, B., \& Goldberg, L. R. (1992). Integration of the Big Five and circumplex approaches to trait structure. Journal of Personality and Social Psychology, 63, 146-163.

Hogan, R. (1969). Development of an empathy scale. Journal of Consulting and Clinical Psychology, 33, 307-316.

Hogan, R. (1983). A socioanalytic theory of personality. In M. M. Page (Ed), Nebraska Simposium on Motivation 1982: PersonalityCurrent theory and research (pp. 55-89). Lincoln: University of Nebraska Press.

Hogan, R. (1986). Hogan Personality Inventory manual. Minneapolis. MN: National Computer Systems.

Hogan, R., \& Hogan, J. (1992). Hogan Personality Inventory manual. Tulsa, OK: Hogan Assessment Systems.

Hogan, R., \& Johnson. J. A. (1979). The Hopkins Personality Inventory: A socioanalytic view of the structure of personality: Unpublished manuscript, Johns Hopkins University, Department of Psychology, Baltimore.

Hogan, R., \& Johnson, J. A. (1981, September). The structure of personality. Paper presented at the 89 th Annual Convention of the American Psychological Association, Los Angeles, CA.

Hogan, R., Johnson, J. A., \& Emler, N. P. (1978). A socioanalytic theory of moral development. In W. Damon (Ed.), New directions for child development: Volume 2. Moral development (pp. 1-18). San Francisco: Jossey-Bass.

John, O. P. (1990a). The "Big Five" factor taxonomy: Dimensions of personality in the natural language and in questionnaires. In L. A. Pervin (Ed.), Handbook of personality theory and research (pp. 66100). New York: Guilford Press.

John, O. P. (1990b). The search for basic dimensions of personality: A review and critique. In P. McReynolds, J. C. Rosen. \& G. L. Chelune (Eds.), Advances in psychological assessment (Vol. 7, pp. 1-37). New York: Plenum Press.

Johnson, J. A. (1983). Criminality, creativity, and craziness: Structural similarities in three types of nonconformity. In W. S. Laufer \& J. M. Day (Eds.), Personality theory, moral development, and criminal behavior (pp. 81-105). Lexington, MA: Heath.

Johnson, J. A. (1991, June). Interpreting the California Psychological Inventory with the $A B 5 C$ model. Invited talk at the University of Groningen, Groningen, the Netherlands.

Johnson, J. A. (1992, June). Multimethod replication of the AB5C model. Paper presented at the Sixth European Conference on Personality, Groningen, the Netherlands.

Johnson, J. A. (in press). Interpreter's guide to the Bipolar Adjective Rating Scales (BARS). Palo Alto, CA: Consulting Psychologists Press.

Johnson, J. A., Cheek, J. M., \& Smither, R. (1983). The structure of empathy. Journal of Personality and Social Psychology. 45, 12991312.

Johnson, J. A., Germer, C. K., Efran, J. S., \& Overton, W. F. (1988).
Personality as the basis for theoretical predilections. Journal of Personality and Social Psychology, 55, 824-835.

Lanning, K., \& Gough, H. G. (1991). Shared variance in the California Psychological Inventory and the California Q-set. Journal of Personality and Social Psychology, 60, 596-606.

Laufer, W. S., Johnson, J. A., \& Hogan, R. (1981). Ego control and criminal behavior. Journal of Personality and Social Psychology, 41, 179-184.

McCrae, R. R. (1987). Creativity, divergent thinking, and openness to experience. Journal of Personality and Social Psychology, 52, 12581265.

McCrae, R. R., \& Costa. P. T., Jr. (1985a). Openness to experience. In R. Hogan \& W. H. Jones (Eds.), Perspectives in personality (Vol. 1, pp. 145-172). Greenwich, CT: JAI Press.

McCrae. R. R., \& Costa, P. T., Jr. (1985b). Updating Norman's "adequate taxonomy": Intelligence and personality dimensions in natural language and in questionnaires. Journal of Personality and Social Psychology: 49, 710-721.

McCrae. R. R.. \& Costa. P. T.. Jr. (1987). Validation of the five-factor model across instruments and observers. Journal of Personality and Social Psychology; 52. 81-90.

McCrae. R. R.. \& Costa. P. T.. Jr. (1989a). Rotation to maximize the construct validity of factors in the NEO Personality Inventory. Multivariate Behavioral Research, 24, 107-124.

McCrae. R. R.. \& Costa. P. T. Jr. (1989b). The structure of interpersonal traits: Wiggins's circumplex and the five-factor model. Journal of Personality and Social Psychology: 56, 586-595.

McCrae. R. R., Costa, P. T., Jr.. \& Piedmont. R. L. (1993). Folk concepts, natural language, and psychological constructs: The California Psychological Inventory and the five-factor model. Journal of Personality and Social Psychology, 61, 1-26.

McCrae, R. R., \& John, O. P. (1992). An introduction to the five-factor model and its applications. Journal of Personality, 60.175-215.

Miller. M. L., Thayer, J. F., \& Johnson, J. A. (1990). The construct validity of openness to experience. Unpublished paper, Pennsylvania State University, Department of Psychology, Dubois. PA.

Norman, W. T. (1963). Toward an adequate taxonomy of personality attributes: Replicated factor structure in peer nomination personality ratings. Journal of Abnormal and Social Psychologl; 66. 574-583.

Ostendorf, F. (1990). Sprache und Persönlichkeitsstruktur: Zur Iáliditat des Fïnf-Faktoren-Modells der Persönlichkeit [Language and personality' structure: Toward the validation of the frve-factormodel of personality]. Regensburg, Germany: S. Roderer Verlag.

Peabody, D., \& Goldberg, L. R. (1989). Some determinants of factor structures from personality-trait descriptors. Journal of Personality and Social Psychology, 57, 552-567.

Rosch. E. (1977). Human categorization. In N. Warren(Ed.). Suclies in cross-cultural psychology (Vol. 1, pp. 1-49). San Diego. CA: Academic Press.

Rosch. E., \& Mervis. C. B. (1975). Family resemblances: Siudies in the internal structure of categories. Cognitive Prycholog! ?, 573-605.

Saucier, G. (1992a). Benchmarks: Integrating affective and interpersonal circles with the Big-Five personality factors. Journal of Personality and Social Psychology: 62.1025-1035.

Saucier. G. (1992b). Openness versus Intellect: Much ado about nothing? European Journal of Personality; 6, 381-386.

Tellegen. A. (1982). Brief manual for the Differ'ntial Pinumativ Questionnaire. Unpublished manuscript, University of Minnesota, Department of Psychology, Minneapolis.

Tellegen, A. (1985). Structure of mood and personality and their relevance to assessing anxiety, with an emphasis on self-report. In A. H. Tuma \& J. D. Maser (Eds.), Anxiety and the anxicty disorders (pp. 681-706). Hillsdale, NJ: Erlbaum. 
Waley, A. (1958). The way and its power: A study of the Tao Tê Ching and its place in Chinese thought. New York: Grove Press.

Watson, D., \& Clark, L. A. (in press). Extraversion and its positive emotional core. In R. Hogan, J. A. Johnson, \& S. R. Briggs (Eds.), Handbook of personality psychology. San Diego, CA: Academic Press.

Weimer, W. B. (1973). Psycholinguistics and Plato's paradoxes of the Meno. American Psychologist, 28, 15-33.

Weimer, W. B. (1984). Limitations of the dispositional analysis of behavior. In J. R. Royce \& L. P. Mos (Eds.), Annals of theoretical psychology (Vol. 1, pp. 161-198). New York: Plenum Press.

Wicklund, R. A. (1990a). Zero-variable theories and the psychology of the explainer. New York: Springer-Verlag.

Wicklund, R. A. (1990b). Zero-variable theories in the analysis of social phenomena. European Journal of Personality, 4, 37-55.
Wiggins, J. S. (1979). A psychological taxonomy of trait-descriptive terms: The interpersonal domain. Journal of Personality and Social Psychology, 37, 395-412.

Wiggins, J. S., \& Pincus, A. L. (1989). Conceptions of personality disorders and dimensions of personality. Psychological Assessment, 1 . 305-316.

Zuckerman, M., Kuhlman, D. M., \& Camac, C. (1988). What lies beyond $\mathrm{E}$ and $\mathrm{N}$ ? Factor analyses of scales believed to measure basic dimensions of personality. Joumal of Personality and Social Psychology, 54, 96-107.

Received December 24, 1992

Revision received February 22, 1993

Accepted February 28, 1993 